\title{
Weak Uniform Distribution for Divisor Functions. I
}

\author{
By Francis J. Rayner
}

\begin{abstract}
Narkiewicz (reference [3, pp. 204-205]) has proposed an algorithm for determining the moduli with respect to which a given arithmetic function (of suitable type) has weak uniform distribution. The class of functions to which this algorithm applies includes the divisor functions $\sigma_{i}$. The present paper gives an improvement to the algorithm for odd values of $i$, which makes computation feasible for values of $i$ up to 200 .

The results of calculations for odd values of $i$ in the range $1 \leq i \leq 199$ are reported.
\end{abstract}

1. Introduction. Let $\sigma_{i}(x)$ be defined for positive integers $i, x$ by

$$
\sigma_{i}(x)=\sum_{d \mid x} d^{i}
$$

For odd values of $i$, the functions $\sigma_{i}$ occur as Fourier coefficients of Eisenstein series.

An arithmetic function $f$ is defined to be weakly uniformly distributed modulo $n$ (WUD $(\bmod n)$, for short) if the set

$$
\{x \in \mathbf{Z}: x>0,(f(x), n)=1\}
$$

is infinite and for every pair of integers $a_{1}, a_{2}$ with $\left(a_{1}, n\right)=\left(a_{2}, n\right)=1$,

$$
\begin{gathered}
\#\left\{x: 0<x<t, f(t) \equiv a_{1} \bmod n\right\} \sim \\
\#\left\{x: 0<x<t, f(x) \equiv a_{1} \bmod n\right\}
\end{gathered}
$$

as $t \rightarrow \infty$.

The integers $n$ for which $\sigma_{i}(x)$ is WUD $(\bmod n)$ have been determined by Sliwa [6] for $i=1$, by Narkiewicz and Rayner [5] for $i=2$, and by Narkiewicz [2] for $i=3$. In the present paper the methods of [2] are further improved. For each odd integer $i>0$, there exist two finite sets of integers $K_{1}$ and $K_{2}$ such that $\sigma_{i}$ has WUD $(\bmod n)$ if and only if either $n$ is odd and not divisible by an element of $K_{1}$ or $n$ is even and not divisible by an element of $K_{2}$.

Calculations of the sets $K_{1}$ and $K_{2}$ for $\sigma_{i}$ for all odd values of $i$ from 5 to 199 have been carried out in the University of Liverpool Computer Laboratory. The results are tabulated at the end of this paper, and the earlier results of Sliwa $(i=1)$ and Narkiewicz $(i=3)$ have been incorporated.

Observation 1. Within the range of the table, it can be seen that if $i$ is prime and $2 i+1$ is composite, then $K_{1}$ is empty, and that if $i$ and $2 i+1$ are both prime, then $K_{1}=\{2 i+1\}$ for $i \equiv 3 \bmod 4$, and $K_{1}=\{6 i+3\}$ for $i \equiv 1 \bmod 4$.

Observation 2. Within the range of the table, if $i$ is prime and $2 i+1$ is composite, then $K_{2}=\{6\}$, with the sole exception of $i=43$, where $K_{2}=\{6,2066\}$. Further, if $i$ is prime and $2 i+1$ is prime, then $K_{2}=\{6,4 i+2\}$.

Received October 11, 1983; revised April 22, 1987.

1980 Mathematics Subject Classification (1985 Revision). Primary 11B99; Secondary 11-04, 11A25, 11F30, 11N69, 11 Y99. 
Observation 3. The upper bound of Lemma 4 below, $(2 i+1)^{2}$, for the set of primes involved in the calculations is much higher than necessary. A value of $(2 i+1)^{1.6}$ would be consistent with the values actually found. It would be possible to make calculations for higher values of $i$ if this observed upper bound could be proved to hold in general.

Since this paper was originally submitted, Narkiewicz's book [4] has appeared. It describes the background and motivation for these calculations and refers to the original version of this paper in which the calculations were carried out for values of $i \leq 107$.

Narkiewicz records that Observation 1 concerning $K_{1}$ has been shown to be true generally by E. Dobrowolski (see [4, p. 110, Theorem 6.12]). (See also Narkiewicz [2] for part of this result.)

In $[4$, p. 112, Problem V] Narkiewicz asks for a characterization of those odd integers $i$ such that $\sigma_{i}$ fails to have WUD $(\bmod n)$ if and only if 6 divides $n$. Since for composite $i$ the set of moduli for which WUD fails is at least the union of the corresponding sets for the factors of $i$, one might first consider prime values for $i$. However, even for prime $i$, there seems to be no easily observed pattern of behavior of $K_{2}$. As in Observation 2 above, in the case in which $i$ is prime and $2 i+1$ is composite, while $K_{1}$ is always empty it is not always true that $K_{2}=\{6\}$, since $\sigma_{43}$ is not WUD (mod 2066), although this seems to be a rare exception. Calculations for prime values of $i$ are easier than for composite ones, and a search beyond the limits of the present tables, assuming a reduced upper bound as in Observation 3, shows that the next primes $i$ for which $K_{2}$ behaves in this way are

$$
\begin{aligned}
& i=467, \quad \text { where } K_{2}=\{6,24286\}, \\
& i=503, \quad \text { where } K_{2}=\{6,24146\}, \text { and } \\
& i=883, \quad \text { where } K_{2}=\{6,38854\} .
\end{aligned}
$$

It is worth noticing in connection with Observation 2 and Dobrowolski's result cited above from [4] that for $i=809$ we have $K_{2}=\{6,3338,38834\}$. Thus, although here $i$ and $2 i+1$ are both prime, it is not always true that under these conditions $K_{2}=\{6,4 i+2\}, 809$ being the first exception.

Because of the reduced upper bound assumed here, these results for $i>200$ may possibly be incomplete in the sense that the sets $K_{2}$ might be larger than stated (and therefore similar results might hold for smaller values of $i$ ), but this is extremely unlikely.

Observation 4. Ramanujan's $\tau$ function has WUD $(\bmod n)$ if and only if either $n$ is odd and not divisible by 7 (Serre) or even and divisible neither by 6 nor 46 (Narkiewicz). (See [4, p. 89, Theorem 5.18].) Thus $\tau$ behaves with respect to weak uniform distribution in the same way as $\sigma_{3}$ for odd $n$ and in the same way as $\sigma_{11}$ for even $n$.

2. Narkiewicz's Algorithm. For a fixed value of $i>2$, let

$$
V_{j}(x)=1+x^{i}+x^{2 i}+\cdots+x^{j i}
$$

Thus, for a prime $p, \sigma_{i}\left(p^{j}\right)=V_{j}(p)$. Let

$$
R_{j}(n)=\left\{V_{j}(a) \bmod n: a \in \mathbf{Z},\left(a V_{j}(a), n\right)=1\right\}
$$


regarded as a subset of the multiplicative group $G(n)$ of residue classes prime to $n$. Let $\Lambda_{j}(n)$ be the subgroup of $G(n)$ generated by $R_{j}(n)$. Let $d(n)$ be the smallest $j \geq 1$ for which $R_{j}(n) \neq \varnothing$.

The following Lemmas 1-4 are special cases of results proved by Narkiewicz [2], [3].

LEMMA 1. $\sigma_{i}$ has $W U D(\bmod n)$ for $i>2$ if and only if $\Lambda_{d(n)}(n)=G(n)$.

Note that for odd $i>2, d(n)=1$ if $n$ is odd, and $d(n)=2$ if $n$ is even. Lemma 1 gives a means of calculating whether $\sigma_{i}$ is WUD $(\bmod n)$ for any particular values of $i$ and $n$.

LEMMA 2. Let $n=q_{1} \cdots q_{r}$, where $q_{1}, \ldots, q_{r}$ are powers of distinct primes. Suppose for each $q_{s}, \Lambda_{j}\left(q_{s}\right)=G\left(q_{s}\right)$. Then $\Lambda_{j}(n) \neq G(n)$ if and only if

(i) there exist characters $\chi_{s}$ of $G\left(q_{s}\right)(s=1, \ldots, r)$ such that $\chi_{s}$ takes a constant value $c_{s}$ (say) on $R_{j}\left(q_{s}\right)$

(ii) $\prod_{s=1}^{r} c_{s}=1$; and

(iii) not all the characters $\chi_{s}$ are trivial.

LEMMA 3. Let $q=p^{t}$, where $p$ is an odd prime. Then there is a nontrivial character of $G(q)$ taking a constant value on $R_{j}(q)$ if and only if there is such a character of $G\left(p^{u}\right)$ taking a constant value on $R_{j}\left(p^{u}\right)$, where $u=\min \{t, 2\}$. For $p=2$ a similar result holds with $u=\min \{t, 3\}$.

LEMMA 4. For any prime $p$, if there is a nontrivial character of $G\left(p^{t}\right)$ taking a constant value on $R_{j}(q)$, then $p<\left(e_{j}+1\right)^{2}$ where $e_{j}$ is the degree of $V_{j}(x)$.

Remark. A slightly stronger result is due to Fomenko [1].

Let $i$ now denote an odd integer greater than 1 . It is easily seen that if $\Lambda_{j}(n) \neq$ $G(n)$, then $\Lambda_{j}(m n) \neq G(m n)$ for any integer $m>1$. It follows that there are finite sets of integers $K_{1}$ and $K_{2}$ such that $\sigma_{i}$ is WUD $(\bmod n)$ if and only if $n$ is odd and not divisible by an element of $K_{1}$ or $n$ is even and not divisible by an element of $K_{2}$. The sets $K_{1}$ and $K_{2}$ can be found in the following way, as follows from Lemmas 1-4.

For $j=1,2$, let $H_{j}$ be the set of primes $p$ satisfying the inequality of Lemma 4 (in which $e_{1}=i$ and $e_{2}=2 i$ ).

Let $I_{j}=H_{j} \cup\left\{p^{2}: p \in H_{j}\right\} \cup\{8\}$, and let

$J_{j}=\left\{m \in I_{j}\right.$ : there exists a nontrivial character on $G(m)$ constant on $\left.R_{j}(m)\right\}$, including cases in which $\Lambda_{j}(m)$ is a proper subgroup of $G(m)$.

Then $K_{j}$ is the set of all products $r$ of elements of $J_{j}$ (no element being taken more than once in each product) for which $\Lambda_{j}(r) \neq G(r)$.

Narkiewicz [2] has determined $K_{1}$ and $K_{2}$ for $i=3$. Because it may be necessary to examine primes $p$ up to $(2 i+1)^{2}$ and to calculate values of $R_{2}\left(p^{2}\right)$ in $G\left(p^{2}\right)$, the calculations become difficult with increasing $i$. The Propositions in Section 3 below make it unnecessary to consider squares of most odd primes and reduce the number of primes which need to be included in the sets $H_{j}$, although the upper bounds are not altered. 
3. Some Improvements. Throughout this paragraph, let $W(x)$ be a polynomial with integer coefficients, and let

$$
R(n)=\{W(a) \bmod n: a \in \mathbf{Z},(a W(a), n)=1\},
$$

regarded as a subset of $G(n)$.

For any prime $q$, let $\phi: G\left(q^{2}\right) \rightarrow G(q)$ be defined, for $x \in \mathbf{Z}$, by $\phi\left(x \bmod q^{2}\right)=$ $x \bmod q$, and let $\psi: G(q) \rightarrow G\left(q^{2}\right)$ be defined, for $x \in \mathbf{Z}$, by $\psi(x \bmod q)=$ $x^{q} \bmod q^{2}$. It is easy to see that $\phi$ and $\psi$ are homomorphisms of abelian groups, that $\psi(\phi(z))=z$ for all $z \in G(q)$ (so that $\phi$ is an epimorphism and $\psi$ is a monomorphism) and that $\phi\left(R\left(q^{2}\right)\right)=R(q)$.

LEMMA 5. Let $\chi$ be any nontrivial character on $G(q)$ which is constant on $R(q)$. Then $\chi \circ \phi$ is a nontrivial character on $G\left(q^{2}\right)$ which is constant on $R\left(q^{2}\right)$.

Proof. Immediate.

LEMMA 6. Let $\chi$ be any nontrivial character on $G\left(q^{2}\right)$ taking the constant value 1 on $R\left(q^{2}\right)$, and suppose that $\chi \circ \psi$ is the trivial character on $G(q)$. Then $R\left(q^{2}\right)$ and $R(q)$ have the same cardinal number.

Proof. First, $R\left(q^{2}\right) \subset \operatorname{ker} \chi$. Again, $\operatorname{im} \psi \subset \operatorname{ker} \chi$. Now $\operatorname{im} \psi$ is a subgroup of $G\left(q^{2}\right)$ of prime index $q$, so, since $\chi$ is not the trivial character, $\operatorname{im} \psi=\operatorname{ker} \chi$. Thus $R\left(q^{2}\right) \subset \operatorname{im} \psi$. The restriction of $\phi$ to $\operatorname{im} \psi$ is bijective, and $\phi\left(R\left(q^{2}\right)\right)=R(q)$. Hence the result.

LEMMA 7. Suppose that the prime number $q$ and polynomial $W(x)$ are such that $\psi(R(q)) \subset R\left(q^{2}\right)$. Let $\chi$ be any nontrivial character on $G\left(q^{2}\right)$ which is constant on $R\left(q^{2}\right)$. Then $\chi \circ \psi$ is a nontrivial character on $G(q)$ which is constant on $R(q)$.

Proof. Since $\psi(R(q)) \subset R\left(q^{2}\right), \chi \circ \psi$ is a character constant on $R(q)$, and it will be enough to show that it is nontrivial. If it is trivial, then $\chi(\psi(R(q)))=1$, and so the constant value of $\chi$ on $R\left(q^{2}\right)$ is 1 . The result now follows from Lemma 6 .

PROPOSITION 1. Let $W(x)=1+x^{i}$, where $i$ is odd and not divisible by the odd prime $q$. Then there is a nontrivial character on $G\left(q^{2}\right)$ constant on $R\left(q^{2}\right)$ if and only if there is a nontrivial character on $G(q)$ constant on $R(q)$.

Proof. It is enough to show that Lemma 7 applies. Let $x \in \mathbf{Z}$ be such that $x \bmod q \neq 0$, and let $y_{\lambda}=x+\lambda q$ for $\lambda=0,1, \ldots, q-1$. Then

$$
\phi\left(\left(1+y_{\lambda}^{i}\right) \bmod q^{2}\right)=\left(1+x^{i}\right) \bmod q
$$

and $1+y_{\lambda}^{i} \equiv 1+y_{\mu}^{i} \bmod q^{2}$ if and only if $\lambda \equiv \mu \bmod q$. Thus $R\left(q^{2}\right)$ contains every element of $G\left(q^{2}\right)$ which is mapped into $R(q)$ by $\phi$. Hence \#R( $\left.q^{2}\right)=q \# R(q)$ and $\psi R(q) \subset R\left(q^{2}\right)$. Since $\psi$ is a monomorphism and $q>2$, Lemmas 5 and 7 now give the result.

Proposition 2. Let $W(x)=1+x^{i}+x^{2 i}$, where $i$ is odd and not divisible by the odd prime $q$. Then there is a nontrivial character on $G\left(q^{2}\right)$ constant on $R\left(q^{2}\right)$ if and only if there is a nontrivial character on $G(q)$ constant on $R(q)$.

Proof. For $q=3$, it is easily seen that such characters exist both on $R(q)$ and on $R\left(q^{2}\right)$. Now suppose $q \geq 5$. It is enough to show that if $\chi$ is a nontrivial character 
on $G\left(q^{2}\right)$ taking a constant value $a$ on $R\left(q^{2}\right)$, then $\chi \circ \psi$ is a nontrivial character on $G(q)$ taking a constant value on $R(q)$. Putting $x=q-1$, we see that $1 \in R\left(q^{2}\right)$, so that $a=\chi(1)=1$. Now let $x$ be such that $x \bmod q \neq 0$, and put $y_{\lambda}=x+\lambda q$ for $\lambda=0,1, \ldots, q-1$. Clearly, $W\left(y_{\lambda}\right) \equiv W\left(y_{\mu}\right) \bmod q^{2}$ if and only if

$$
(\lambda-\mu) i x^{i-1}\left(1+2 x^{i}\right) \equiv 0 \bmod q .
$$

If $x$ is such that $1+2 x^{i} \bmod q \neq 0$, it follows that $q$ distinct elements of $R\left(q^{2}\right)$ are mapped onto $W(x) \bmod q$ by $\phi$. On the other hand, if $x$ is such that $1+2 x^{i} \bmod q=$ 0 , then exactly one element of $R\left(q^{2}\right)$ is mapped onto $W(x) \bmod q$ by $\phi$. Note that in this case $W(x) \bmod q$ is uniquely determined. Thus, provided $R(q)$ has at least two elements, we can conclude that $\# R\left(q^{2}\right)>\# R(q)$. But $q$ is a prime greater than 3 , and $1 \in R(q), 3 \in R(q)$. Lemma 6 now shows that $\chi \circ \phi$ is nontrivial. Now let $z \bmod q$ be any element of $R(q)$, so that $z=W(x) \bmod q$ for suitable $x \in \mathbf{Z}$. Then $z \bmod q^{2} \in R\left(q^{2}\right)$, and

$$
\chi(\phi(z \bmod q))=\chi\left(z^{q} \bmod q^{2}\right)=\left(\chi\left(z \bmod q^{2}\right)\right)^{q}=1^{q}=1 .
$$

Thus $\chi \circ \phi$ is constant on $R(q)$, and the proposition is proved.

Proposition 3. Let $i$ be odd, and let $q$ be a prime greater than 3 , and let $W(x)$ be either $1+x^{i}$ or $1+x^{i}+x^{2 i}$. Suppose that there is a nontrivial character on $G(q)$ which is constant on $R(q)$. Then $(i, q-1) \neq 1$.

Proof. Suppose that $(i, q-1)=1$. Then $x \rightarrow x^{i}$ is an automorphism of $G(q)$.

For $W(x)=1+x^{i}$ we have $R(q)=\{2,3, \ldots, q-1\}$ and the only character constant on this set is trivial, so that the proposition holds in this case.

For $W(x)=1+x^{i}+x^{2 i}=\left(x^{i}+\alpha\right)^{2}+\beta$, where $\alpha$ and $\beta$ are calculated in the finite field $\mathbf{Z}_{q}$, we have $1=W(-1) \in R(q)$, so that there will only be a nontrivial character constant on $R(q)$ if $R(q)$ generates a proper subgroup of $G(q)$. As $x^{i}$ runs through all the nonzero elements of $\mathbf{Z}_{q}, x^{i}+\alpha$ runs through all except $\alpha$ (but including 0 and $-\alpha)$, so that $\left(x^{i}+\alpha\right)^{2}$ runs through all the quadratic residues, and also takes the value 0 . Thus $\left(x^{i}+\alpha\right)^{2}+\beta$ takes $(q-1) / 2$ values in $G(q)$ if $-\beta$ is a quadratic residue, and $(q+1) / 2$ values otherwise. If $R(q)$ generates a proper subgroup of $G(q)$, this can only be the subgroup of order $(q-1) / 2$, that is, the group of quadratic residues. Thus, for every quadratic residue $r^{2}, r^{2}+\beta$ is also a quadratic residue. It follows that every element of $G(q)$ is a quadratic residue. This contradiction completes the proof of the proposition.

4. Results. With the help of Propositions 1, 2 and 3, the algorithm of Section 2 can be simplified as follows.

For an odd integer $i>1$, let $H_{1}$ (respectively, $H_{2}$ ) be the set consisting of the primes $p$ of the form $1+\lambda r$ (where $r$ is a nontrivial divisor of $i$ and $\lambda$ is an integer) for which $p<(i+1)^{2}$ (respectively, $p<(2 i+1)^{2}$ ), together with the prime divisors of $i$ and their squares.

Let

$$
I_{1}=H_{1} \cup\left\{p^{2}: p \in H_{1} \text { is prime and there exists } q \in H_{1} \text { with } q \equiv 1(\bmod p)\right\}
$$

and let

$$
\begin{aligned}
I_{2}= & H_{2} \cup\left\{p^{2}: p \in H_{2} \text { is prime and there exists } q \in H_{2} \text { with } q \equiv 1(\bmod p)\right\} \\
& \cup\{2,4,8\} .
\end{aligned}
$$


As before, let $J_{1}$ be the subset of $I_{1}$ consisting of those elements $m$ for which there is a nontrivial character modulo $m$ constant on the set $R(m)$ of values of the polynomial $1+x^{i}$, and let $J_{2}$ be calculated similarly from $I_{2}$ using $1+x^{i}+x^{2 i}$. The sets $K_{1}$ and $K_{2}$ consist of the products $r$ (say) of elements of $J_{1}$ and $J_{2}$, respectively, with no repeated factor, for which $\Lambda_{1}(r) \neq G(r)$ (respectively, $\Lambda_{2}(r) \neq G(r)$ ), but omitting from $K_{1}$ and $K_{2}$ any $r$ which is strictly divisible by another element already known to lie in $K_{1}$ or $K_{2}$, respectively. It follows from the results of Section 3 that, with $K_{1}$ and $K_{2}$ found from these smaller sets $I_{1}$ and $I_{2}, \sigma_{i}$ fails to have WUD $(\bmod n)$ if and only if $n$ is odd and divisible by an element of $K_{1}$ or $n$ is even and divisible by an element of $K_{2}$.

The results tabulated below include the cases $i=1$, due to Sliwa [6] and $i=3$ due to Narkiewicz [2].

\section{TABLE OF RESULTS}

The notation is as in Section 2. $\sigma_{i}$ has WUD $(\bmod n)$ if and only if $n$ is odd and not divisible by an element of $K_{1}$ or $n$ is even and not divisible by any element of $K_{2}$.

\begin{tabular}{|c|c|c|}
\hline$i$ & $K_{1}$ & $K_{2}$ \\
\hline 1 & - & 6 \\
\hline 3 & 7 & 6 \\
\hline 5 & 33 & 622 \\
\hline 7 & - & 6 \\
\hline 9 & 757 & 6146 \\
\hline 11 & 23 & 646 \\
\hline 13 & - & 6 \\
\hline 15 & 73133 & 622122302 \\
\hline 17 & - & 6 \\
\hline 19 & - & 6 \\
\hline 21 & 743 & 6 \\
\hline 23 & 47 & 694 \\
\hline 25 & 33 & 622 \\
\hline 27 & 757109 & 6146 \\
\hline 29 & 177 & 6118 \\
\hline 31 & - & 6 \\
\hline 33 & 723201 & 646134 \\
\hline 35 & 3371 & 622142 \\
\hline 37 & - & 6 \\
\hline 39 & 779157 & 61874 \\
\hline 41 & 249 & 6166 \\
\hline 43 & - & 62066 \\
\hline 45 & 7313357209 & 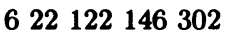 \\
\hline 47 & - & 6 \\
\hline 49 & - & 6 \\
\hline 51 & 7103307 & 6206614 \\
\hline 53 & 321 & 6214 \\
\hline 55 & 2333 & 62246 \\
\hline 57 & 7229 & 6 \\
\hline 59 & - & 6 \\
\hline
\end{tabular}




\begin{tabular}{|c|c|c|}
\hline 61 & - & 6 \\
\hline 63 & $74357 \quad 127$ & 6146 \\
\hline 65 & 333931441 & 622262 \\
\hline 67 & - & 6 \\
\hline 69 & 747277417 & 694 \\
\hline 71 & - & 6 \\
\hline 73 & - & 6 \\
\hline 75 & 73133151 & $\begin{array}{lllll}622 & 122 & 302 & 1202 & 2402\end{array}$ \\
\hline 77 & 23 & 646 \\
\hline 79 & - & 6 \\
\hline 81 & $\begin{array}{lllll}7 & 57 & 109 & 489 & 3097\end{array}$ & 6146 \\
\hline 83 & 167 & 6334 \\
\hline 85 & 33 & 6223742 \\
\hline 87 & 7177 & 6118 \\
\hline 89 & 537 & 6358 \\
\hline 91 & - & 6 \\
\hline 93 & 7 & 6 \\
\hline 95 & 33191 & 622382 \\
\hline 97 & - & 6 \\
\hline 99 & 723571992013971273 & 646134146 \\
\hline 101 & - & 6 \\
\hline 103 & 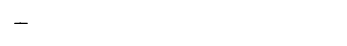 & 6 \\
\hline 105 & 7313343716332321 & $\begin{array}{lllll}622 & 122 & 142 & 302\end{array}$ \\
\hline 107 & - & 6 \\
\hline 109 & - & 6 \\
\hline 111 & 7223 & 6 \\
\hline 113 & 681 & 6454 \\
\hline 115 & 3347 & 62294 \\
\hline 117 & 75779157 & 61461874 \\
\hline 119 & 239 & 6478 \\
\hline 121 & 23 & 646 \\
\hline 123 & 7249 & 6166 \\
\hline 125 & 33251 & 622502 \\
\hline 127 & - & 6 \\
\hline 129 & 7 & 62066 \\
\hline 131 & 263 & 6526 \\
\hline 133 & - & 6 \\
\hline 135 & $\begin{array}{llllll}731 & 33 & 57 & 109 & 209 & 271\end{array}$ & 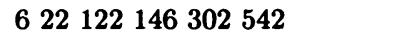 \\
\hline 137 & - & 6 \\
\hline 139 & - & 6 \\
\hline 141 & 7283 & 6 \\
\hline 143 & 23 & 646 \\
\hline 145 & 33177649 & 622118 \\
\hline 147 & 743 & 6 \\
\hline 149 & - & 6 \\
\hline 151 & - & 6 \\
\hline 153 & 757103307919 & 6146206614122618387346 \\
\hline 155 & 33311 & 622622 \\
\hline 157 & - & 6 \\
\hline 159 & 7321 & 6214 \\
\hline
\end{tabular}


(continued)

$\begin{array}{lllll}161 & 47 & 694 \\ 163 & - & 6 & \\ 165 & 7233133201331737 & 622461221343021322 \\ 167 & - & 6 & \\ 169 & - & 6 & \\ & & & \\ 171 & 757229 & 6146 \\ 173 & 1041 & 6694 \\ 175 & 3371 & 622142 \\ 177 & 7 & 6 & 6718 \\ 179 & 359 & \\ & & 6 & \\ 181 & - & 6734 \\ 183 & 7367733 & 622 \\ 185 & 33 & 646 \\ 187 & 23 & 61461514 \\ 189 & 7435710912711377201 & \\ & & 6766 \\ 191 & 383 & 6 & \\ 193 & - & 6221222623021874 \\ 195 & 73133791573931441 & 6 & \\ 197 & - & 6\end{array}$

Department of Pure Mathematics

The University of Liverpool

P.O. Box 147

Liverpool, Great Britain GB-L69 3BX

1. O. M. FoMENKo, "The distribution of values of multiplicative functions with respect to a prime modulus," Zap. Nauchn. Sem. Leningrad. Otdel. Mat. Inst. Steklov. (LOMI), v. 93, 1980, pp. 218-224. (Russian)

2. W. NARKIEWICZ, "Distribution of coefficients of Eisenstein series in residue classes," Acta Arith., v. 43, 1983, pp. 83-92.

3. W. NARKIEWICZ, "Euler's function and the sum of divisors," J. Reine Angew. Math., v. 323, 1981, pp. 200-212.

4. W. NARKIEWICZ, Uniform Distribution of Sequences of Integers in Residue Classes, Lecture Notes in Math., vol. 1087, Springer-Verlag, Berlin and New York, 1984.

5. W. NARKIEWICZ \& F. RAYNER, "Distribution of values of $\sigma_{2}(n)$ in residue classes," Monatsh. Math., v. 94, 1982, pp. 133-141.

6. J. SLIWA, "On distribution of values of $\sigma(n)$ in residue classes," Colloq. Math., v. 28, 1973, pp. 283-291. 